\author{
Maria Alice FUSCO ${ }^{1}$ \\ João TELHADO \\ Jorge da Silva PEREIRA ${ }^{3}$ \\ Fernanda Guimarães \\ JANUZZI ${ }^{3}$ \\ Paulo de Tarso Landgraf \\ BOTTEON $^{2}$
}

Correspondência para:

Maria Alice Fusco, Rua Bom Retiro, 165/

201, Ilha do Governador, Rio de Janeiro,

Brasil, 021940-320,mafvet@yahoo.com.br

Recebido para publicação: 09/02/2007 Aprovado para publicação: 27/08/2009

\title{
Meloxicam e prednisona: efeitos do tratamento oral de curto prazo sobre os níveis de pressão intra- ocular em cães
}

\author{
1 - Doutoranda do Curso de Pós-Graduação em Medicina da Universidade \\ Federal do Rio de Janeiro, Rio de Janeiro-RJ \\ 2 - Departamento de Medicina e Cirurgia Veterinária da Universidade \\ Federal Rural do Rio de Janeiro, Seropédica-RJ \\ 3 - Centro de Estudos, Pesquisa e Oftalmologia Veterinária, Rio de Janeiro-RJ
}

\section{Resumo}

Para possível observação da hipertensão ocular com o uso de antiinflamatórios, foram selecionados 28 cães da raça Beagle. Para avaliação da pressão intra-ocular antes do tratamento, no dia 0 (zero) todos os animais tiveram a pressão intra-ocular avaliada às 08 horas e às 16 horas. No dia seguinte dez cães receberam meloxicam na dose de $0,2 \mathrm{mg} / \mathrm{kg}, \mathrm{e} 0,1 \mathrm{mg} / \mathrm{kg}$ nos restantes quatro dias. Nove cães receberam prednisona na dose de $1,0 \mathrm{mg} / \mathrm{kg}$ durante cinco dias. Nove cães receberam somente porção de ração úmida. No quinto dia do tratamento todos os animais tiveram novamente a pressão intraocular avaliada às 08 horas e às 16 horas. Em todos os grupos, incluindo o grupo-controle, as maiores médias de pressão intra-ocular foram observadas no dia 5 (cinco). A diferença dos valores de pressão intra-ocular observada entre as medições das 08 horas e das 16 horas foi significativa, independente do tratamento e do dia considerado. $\mathrm{O}$ uso dos anti-inflamatórios esteroidal e não-esteroidal não foi capaz de causar hipertensão ocular e alguns fatores podem ser incriminados, como via de administração, dose e duração do tratamento utilizado, além da ausência de doença glaucomatosa nos cães selecionados.

\author{
Palavras-chave: \\ Cão. \\ Hipertensão ocular. \\ Anti-inflamatório não-esteroidal. \\ Glicocorticóide
}

\section{Introdução}

O humor aquoso contido no olho exerce pressão contra a parede do bulbo ocular com uma força que é denominada pressão intra-ocular (PIO). ${ }^{1}$ A hipertensão ocular constitui um fator de risco para o desenvolvimento de glaucoma ${ }^{2,3,4,5}$, sendo este fator o único passível de tratamento ${ }^{3,6}$. O glaucoma é definido como uma neuropatia óptica anterior progressiva caracterizada por escavação e atrofia do disco óptico e decréscimo na funcionalidade da retina ${ }^{2,7,8}$, geralmente acompanhada por valores de PIO maiores que $25 \mathrm{mmHg}^{9}$. Sendo os danos irreversíveis e tendo a cegueira como resultado final, o diagnóstico e tratamento precoces seriam formas eficientes de retardar o seu aparecimento. ${ }^{10}$
Descobertas recentes nos levam à hipótese de que o uso de anti-inflamatórios esteroidais (AIE) e não-esteroidais (AINE) apresente, como efeito colateral, a elevação da PIO. A primeira refere-se ao efeito hipotensor ocular das prostaglandinas e à introdução das prostaglandinas sintéticas entre as opções para o controle medicamentoso do glaucoma. ${ }^{11,12}$ Outra observação mais recente ${ }^{13}$ refere-se à expressão de COX-2 de forma constitutiva no segmento anterior do olho humano, e à diminuição ou ausência desta enzima no epitélio não-pigmentado do corpo ciliar em olhos portadores de glaucoma de ângulo aberto ou induzido por corticosteróides; nestes olhos observou-se, ainda, a baixa concentração de prostaglandinas no humor aquoso em comparação com os olhos 
normais. Como os AIEs e os AINEs atuam inibindo a expressão de COX-2 $2^{13,14,15} \mathrm{e}$, consequentemente, a produção de prostaglandinas ${ }^{15}$, estes fármacos podem ter algum efeito na pressão intra-ocular.

A hipertensão ocular como resposta ao uso de anti-inflamatórios esteroidais em pacientes humanos adultos vem sendo descrita a algumas décadas ${ }^{16,17,18}$, sendo o glaucoma um efeito colateral dos glicocorticóides exógenos em humanos ${ }^{19}$. Este efeito pode ocorrer independentemente da dose, dose total, duração do tratamento, fármaco ${ }^{20}$, via de administração $\mathrm{O}^{19,20,21,22} \mathrm{e}$, quanto maior a capacidade anti-inflamatória do AIE, maior a probabilidade de elevação da $\mathrm{PIO}^{19,21}$. Entretanto, mesmo após anos de conhecimento sobre a relação entre o uso de antiinflamatórios esteroidais e o aumento da pressão intra-ocular, a patogênese desta desordem carece de maiores esclarecimentos. ${ }^{23}$

$\mathrm{Na}$ medicina veterinária, são poucos os relatos de hipertensão ocular associada ao uso de anti-inflamatórios esteroidais. ${ }^{24,25,26,27}$

Devido aos efeitos colaterais ensejados pelo uso prolongado dos AIEs sistêmicos, os AINEs são uma alternativa na terapia antiinflamatória, tornando-se bastante popular a prescrição desta classe de medicamentos por médicos veterinários. Os AINEs possuem, além dos efeitos anti-inflamatórios, efeitos analgésico e antipirético. São comumente utilizados no tratamento da dor nas doenças músculo-esqueléticas. ${ }^{28} \mathrm{Na}$ medicina veterinária o meloxicam é um dos AINEs mais utilizados pela via oral, de escolha para o controle da dor crônica e da inflamação em pequenos animais. $^{29}$

Objetivou-se, neste estudo, a observação da variação da pressão intraocular em cães tratados, por via oral, com prednisona (AIE) ou meloxicam (AINE).

\section{Material e Método}

O experimento descrito a seguir teve aprovação da Comissão de Ética na Pesquisa da Universidade Federal Rural do Rio de Janeiro (UFRRJ - Protocolo $\left.\mathrm{n}^{\circ} 021 / 2009\right)$.

Foram selecionados e utilizados 28 cães (Canis familiares) da raça Beagle, machos e fêmeas não castrados, com idade variando de seis meses a seis anos, peso de oito a 20 quilos, sem alterações clínicas oculares ou sistêmicas, pertencentes ao Canil do Laboratório de Desenvolvimento de Produtos Parasiticidas do Departamento de Parasitologia Animal do Instituto de Veterinária da UFRRJ. A alimentação consistiu de ração seca industrializada e água fresca ad libitum.

Os 28 cães foram distribuídos, de forma aleatória, entre três grupos de tratamentos A, B e C, que receberam:Grupo A - meloxicam $\left(\right.$ Meloxivet $^{\circledR}$, Duprat, Rio de Janeiro, RJ), misturado a algumas porções de ração úmida industrializada; Grupo B prednisona (Meticorten Veterinário ${ }^{\circledR}$, Schering-Plough, Cotia, SP) misturado a algumas porções de ração úmida industrializada e Grupo C - algumas porções de ração úmida industrializada (grupo controle).

No dia 0 (zero) todos os 28 animais tiveram a PIO mensurada com o uso do tonômetro de aplanação (TonoPen ${ }^{\circledR}$, Reichert, Ohio, EUA) às $8 \mathrm{~h}$ e às $16 \mathrm{~h}$, para o registro dos valores basais.

No dia 1 (hum) todos os dez cães pertencentes ao grupo A receberam meloxicam por via oral, misturado com duas ou três porções de ração úmida, na dosagem de $0,2 \mathrm{mg} / \mathrm{kg}$, às $8 \mathrm{~h}$, segundo protocolos de Teixeira ${ }^{15}$ e Giuliano $^{30}$. Ainda neste dia, os cães do grupo B receberam prednisona por via oral, misturado com duas ou três porções de ração úmida, na dosagem de $1,0 \mathrm{mg} / \mathrm{kg}$, às 8 h. Os cães do grupo $\mathrm{C}$, por sua vez, receberam somente duas ou três porções de ração úmida no mesmo horário. Nos dias 2 (dois), 3 (três) e 4 (quatro), todos os cães do grupo A receberam meloxicam por via oral, misturado com duas ou três porções de ração úmida, na dose de $0,1 \mathrm{mg} / \mathrm{kg}$, às 8h. ${ }^{15,30}$ Os cães do grupo B receberam prednisona por via oral, misturado com duas ou três porções de ração úmida, na dose de $1,0 \mathrm{mg} / \mathrm{kg}$, às $8 \mathrm{~h}$, e os cães do grupo C 
receberam somente duas ou três porções de ração úmida nestes horários. No dia 5 (cinco) os cães do grupo A tiveram a pressão intraocular avaliada às $8 \mathrm{~h}$ e, ato contínuo, receberam meloxicam por via oral, misturado com duas ou três porções de ração úmida, na dose de $0,1 \mathrm{mg} / \mathrm{kg} .^{15,30}$ Ainda neste dia, os cães do grupo B também tiveram a pressão intra-ocular avaliada às $8 \mathrm{~h}$ e, a seguir, receberam prednisona por via oral, misturado com duas ou três porções de ração úmida, na dosagem de $1,0 \mathrm{mg} / \mathrm{kg}$. Os animais do grupo $\mathrm{C}$ tiveram a pressão intra-ocular avaliada e, logo a seguir, lhes era ofertado somente ração úmida. Às $16 \mathrm{~h}$ do dia 5 (cinco), todos os 28 cães tiveram a pressão intra-ocular novamente avaliada.

\section{Resultados}

O resumo dos valores médios de pressão intra-ocular mensurados às $8 \mathrm{~h}$ e às $16 \mathrm{~h}$ dos dias 0 (zero) e 5 (cinco) dos grupos A, B e C encontram-se na tabela 1.

Para comparar o comportamento dos grupos ao longo do tempo, considerando-se a relação existente entre medidas tomadas em um mesmo animal, foi empregado o modelo de análise de variância com medidas repetidas ${ }^{31}$. Considerando o nível de significância $=0,05$, os resultados obtidos permitem afirmar que os valores médios das pressões intraoculares entre grupos não variaram significativamente $(\mathrm{p}=0,810)$; os valores médios aferidos no dia 0 e no dia 5 diferiram significativamente $(p=0,008)$ em todos os grupos, incluindo aí o grupo $\mathrm{C}$ que não recebeu tratamento medicamento; esta diferença verificada foi a mesma nos três grupos ( $p=0,913)$; a diferença da pressão intra-ocular média observada entre as avaliações das 08 horas e das 16 horas foi significativa $(\mathrm{p}=0,001)$; a diferença observada independe do dia considerado $(\mathrm{p}$ $=0,291)$, e foi a mesma nos três grupos $(\mathrm{p}$ $=0,678)$.

\section{Discussão}

O uso do Tono-Pen ${ }^{\circledR}$ é justificado pelo fato deste aparelho proporcionar maior fidedignidade na avaliação da PIO em cães. ${ }^{32}$

A avaliação da PIO no dia 0 (zero) visou a observação dos valores basais para posterior comparação com os valores de PIO apresentados após os tratamentos. A avaliação da pressão intra-ocular no dia 5 (cinco) foi realizada para se identificar um possível efeito aditivo do uso do meloxicam após o período de cinco dias de tratamento, período este comumente de escolha na prática veterinária. ${ }^{15,30} \mathrm{~A}$ avaliação da $\mathrm{PIO}$ às $16 \mathrm{~h}$ visou buscar um possível efeito imediato, oito horas após administração do medicamento, visto ser este o tempo em que a concentração plasmática do meloxicam é máxima. ${ }^{29} \mathrm{O}$ tratamento com prednisona objetivou uma possível comparação de efeitos entre o AINE e o AIE nos níveis da

Tabela 1 - Médias e desvios-padrão dos valores de pressão intra-ocular, em mmHg, em 28 cães Beagle avaliados com o uso do Tono-Pen ${ }^{\circledR}$, às 8 horas e às 16 horas do dia 0 (zero) - sem tratamento - e às 8 horas e às 16 horas do dia 5 (cinco) - após cinco dias de tratamento, nos grupos A (meloxicam), B (prednisona) e C (sem tratamento medicamentoso). Seropédica -RJ, 2006

\begin{tabular}{lccccc}
\hline & & \multicolumn{2}{c}{ Dia 0 } & \multicolumn{2}{c}{ Dia 5 } \\
\cline { 3 - 6 } & & $8 \mathrm{~h}$ & $16 \mathrm{~h}$ & $8 \mathrm{~h}$ & $16 \mathrm{~h}$ \\
\hline \multirow{2}{*}{ Grupo A } & Média & 16,7 & 14,5 & 17,7 & 15,8 \\
& Desvio-padrão & 1,6 & 2,3 & 2,6 & 1,3 \\
\hline \multirow{2}{*}{ Grupo B } & Média & 15,9 & 14,9 & 17,5 & 15,4 \\
& Desvio-padrão & 1,5 & 1,9 & 2,1 & 1,8 \\
\hline \multirow{2}{*}{ Grupo C } & Média & 16,2 & 15,6 & 17,6 & 15,8 \\
& Desvio-padrão & 1,8 & 2,1 & 2,5 & 2,2 \\
\hline
\end{tabular}


PIO, com base no conhecimento a respeito do efeito hipertensivo ocular com o uso de AIE. $16,17,18,19$

Todos os grupos apresentaram valores médios de PIO dentro dos limites considerados seguros ${ }^{9}$, em todas as avaliações (Tabela 1). Os valores da PIO nos grupos $\mathrm{A}, \mathrm{B}$ e $\mathrm{C}$ não diferiram, em média, ao longo das avaliações tomadas nos dois horários e entre os dias 0 (zero) e 5 (cinco).

As maiores médias de PIO foram observadas às $8 \mathrm{~h}$, em todos os grupos, concordando com Gelatt e MacKay ${ }^{32}$, Gelatt e Gum $^{33}$, Heat ${ }^{34}$ e Slatter ${ }^{35}$ a respeito do ritmo circadiano da PIO.

Entretanto, nos três grupos, as maiores médias de PIO foram observadas no dia 5 (cinco), incluindo o grupo C, que não recebeu qualquer tratamento. Este resultado interessante também foi observado por Herring e Ward ${ }^{17}$ em seu grupo-controle no estudo para se determinar o efeito da administração oral de hidrocortisona sobre a PIO de cães normotensos.

Com as informações coletadas na revisão de literatura, esperava-se que as administrações aos cães dos anti-inflamatórios meloxicam e prednisona ensejariam hipertensão ocular, o que não ocorreu.

Em relação à prednisona, sabe-se que a ocorrência de hipertensão associada ao uso de AIE em humanos por via sistêmica é pequena. ${ }^{23}$ Além da via de administração, outros fatores também são importantes no aparecimento da hipertensão ocular e do glaucoma, como dose, freqüência diária, duração do tratamento e potência do AIE utilizado. Renfro e Snow ${ }^{21}$ e Johnson et al. ${ }^{23}$ citaram também a pré-existência do glaucoma como fator para observação do aumento da pressão com o uso de AIE. Gelatt e Mackay ${ }^{26}$ observaram aumento da PIO após uso tópico de dexametasona, em cães da raça Beagle com diagnóstico prévio de glaucoma.

Em relação ao meloxicam, este AINE não possui uma maior seletividade para COX-2 em cães ${ }^{36,37}$ apesar desta crença ainda presente em nosso meio profissional. Portanto, a ausência do fenômeno de hipertensão ocular pode ser o resultado de sua pouca seletividade na inibição desta isoenzima presente nos tecidos oculares. A escolha da administração do meloxicam, por via oral nesta dose e duração do tratamento, também pode ter influência na resposta na pressão intra-ocular, entretanto a falta de publicações sobre este assunto dificulta o cotejamento.

Os autores visaram, utilizando o meloxicam por via oral durante cinco dias, mimetizar a indicação de uso do anti-inflamatório não-esteroidal rotineiramente aplicada na prática veterinária. ${ }^{15,29,30} \mathrm{~A}$ escolha da prednisona baseou-se na revisão de literatura a respeito do efeito hipertensivo ocular dos corticóides ${ }^{16,17,18,19,20,21,22}$ e para efeito de comparação com o grupo tratado com o meloxicam, o corticóide foi administrado pela mesma via de administração e utilizado pelo mesmo período de tratamento.

Apesar de não ter sido observada a hipertensão ocular induzida por antiinflamatórios (esteroidais e não-esteroidais) nos cães do nosso trabalho, relatos deste fenômeno tanto em humanos quanto em animais suscita a importância do monitoramento da pressão intra-ocular por meio da tonometria ${ }^{38}$ nos cães submetidos a qualquer tratamento anti-inflamatório, em especial naquelas raças sabidamente predispostas, como Basset Hound, Beagle, Cocker Spaniel e Poodle ${ }^{38}$. Por se tratar de um evento relacionado ao risco de desenvolvimento do glaucoma $\mathrm{a}^{2,3,4,5,38}$ e sendo o glaucoma considerado uma das causas mais comuns de cegueira nos cães ${ }^{38,39}$, a hipertensão ocular deve ser tratada. Para tal objetivo são utilizados os medicamentos antiglaucomatosos, confeccionados para diminuir a pressão intra-ocular ${ }^{39}$. Por não ser o escopo do presente trabalho, sugerimos a leitura de Ribeiro, Martins e Laus ${ }^{39}$ a respeito dos principais medicamentos hipotensores tópicos disponíveis.

\section{Conclusões}

O uso do meloxicam por via oral a 
cada 24 horas, na dose de $0,2 \mathrm{mg} / \mathrm{kg}$ no primeiro dia e $0,1 \mathrm{mg} / \mathrm{kg}$ nos quatro dias subseqüentes não causa hipertensão intraocular em cães da raça Beagle normotensos. Também o uso da prednisona por via oral a cada 24 horas na dose de $1,0 \mathrm{mg} / \mathrm{kg}$ durante cinco dias não causa hipertensão intra-ocular em cães da raça Beagle normotensos. O fato de não ter sido desenvolvida a hipertensão ocular neste estudo não exclui a possibilidade de observação do evento hipertensivo com a utilização de outro regime de administração, dose e duração do tratamento. Também não se exclui a possibilidade de aumento da pressão intra-ocular com o tratamento antiinflamatório em cães glaucomatosos.

\section{Agradecimentos}

Ao Prof. Dr. Fábio Barbour Scott, responsável pelo Canil do Laboratório de Desenvolvimento de Produtos Parasiticidas - Departamento de Parasitologia Animal Instituto de Veterinária - Universidade Federal Rural do Rio de Janeiro.

\title{
Meloxican and prednisone: the effects of orally short term treatment on the intra-ocular pressure levels in dogs
}

\begin{abstract}
In order to observe a possible ocular hypertension associated with the use of anti-inflammatory drugs, 28 beagle dogs were selected. For evaluation of intraocular pressure before treatment, the totality of animals had their intraocular pressure measured at 8 a.m. and 4 p.m. on day 0 (zero). On the following day 10 animals received meloxican on dose of $0.2 \mathrm{mg} / \mathrm{Kg}$ and $0.1 \mathrm{mg} / \mathrm{Kg}$ on the four remaining days. Nine dogs received prednisone on dose of $1,0 \mathrm{mg} / \mathrm{Kg}$ during five days. Nine dogs received only wet feeding. On the fifth day of treatment the totality of dogs had their intraocular pressure measured at 8 a.m. and 4 p.m. For all groups, including control-group, the highest average values of intraocular pressure were observed on day 5 (five). The difference between the two evaluations of intra-ocular pressure $(8$ a.m. and 4 p.m) was significant, independent of treatment and of the considered day. The use of both steroidal or non-steroidal antiinflammatory were not capable of causing ocular hypertension and some factors can be pointed out, such as route of administration, dosage and duration of therapy chosen, besides absence of glaucomatous disorder between the selected dogs.
\end{abstract}

\section{Referências}

1 BROSS-SORIANO, D.; HANENBERG-MILVER,C; SCHIMELMITZ-IDI, J.; ARRIETA-GOMEZ,J. R.; DEL TORO, R. A.; BRAVO-ESCOBAR, G.. Effects of three nasal topical steroids in the intraocular pressure compartment. Archives of Otolaryngology-Head \& Neck Surgery, v. 130, n. 2, p. 187-191, 2004.

2 HASHIMOTO, M.; SILVA, M. R. B. M.; TEIXEIRA NETO, F. J. Efeito de drogas utilizadas no tratamento de hipertensão arterial sistêmica sobre a pressão intraocular: estudo experimental no cão. Arquivos Brasileiros de Oftalmologia, v. 65, n. 2, p. 229-233, 2002. Disponível em: < http://www.scielo.br/pdf/abo/v65n2/ 9557.pdf >. Acesso em: 24 jul. 2008.
Key words:

Dog.

Ocular hypertension.

Non-steroidal anti-inflammatory. Glucocorticoid
3 WILLIS, A. M.; DIEHL, K. A.; ROBBIN, T. E. Advances in topical glaucoma therapy. Veterinary Ophthalmology, v. 5, n. 1, p. 9-17, 2002.

4 GOLDBERG, I. Relationship between intraocular pressure and preservation of visual field in glaucoma. Survey of Ophthalmology, v. 48, p. S3-S7, 2003. Supplement 1.

5 GUO, L.; MOSS, S. E.; ALEXANDER, R. A.; ALI, R. R.; FITZE, F. W.; CORDEIRO, M. F. Retinal ganglion cell apoptosis in glaucoma is related to intraocular pressure and iop-induced effects on extracellular matrix. Investigative Ophthalmology \& Visual Science, v. 46, n. 1, p. 175-182, 2005.

6 TINGEY, D.; BERNARD, L. M.; GRIMA, D. T.; 
MILLER, B.; LAM, A. Intraocular pressure control and persistence on treatment in glaucoma and ocular hypertension. Canadian Journal of Ophthalmology, v. 40, n. 2, p. 161-169, 2005. Disponível em: <http:// pubs.nrc-cnrc.gc.ca/cjo/cjo40/i05-032.pdf > . Acesso em: 24 jul. 2008.

7 CVENKEL, B. Retinal thickness at the posterior pole in glaucoma and ocular hypertension. Graefes Archive for Clinical and Experimental Ophthalmology, v. 242, n. 11, p. 920-925, 2004.

8 RUIZ-EDERRA, J.; VERKMAN, A. S. Mouse model of sustained elevation in intraocular pressure produced by episcleral vein occlusion. Experimental Eye Research, v. 82, n. 5 , p. $879-84,2005$.

9 MILLER, P. E. Glaucoma: diagnosis and therapy. In: ANNUAL WALTHAM/OSU SYMPOSIUM, SMALL ANIMAL OPHTHALMOLOGY, 25., 2001, Ohio. [Procedings...]. 2001. 14 p. Disponível em: <http:// www.vin.com/VINDBPub/SearchPB/Proceedings/ PR05000/PR00522.pdf >. Acesso em: 24 jul. 2008.

10 MAGACHO, L.; MARCONDES, A. M.; COSTA, V. $P$. Discriminação entre olhos normais e glaucomatosos mediante polarimetria de varredura a laser. Arquivos Brasileiros de Oftalmologia, v. 67, n. 2, p. 249-254, 2004. Disponível em: < http://www.scielo.br/pdf/abo/ v67n2/19749.pdf >. Acesso em: 24 jul. 2008.

11 BITO, L. Z. A new approach to the medical management of glaucoma, from the bench to the clinic, and beyond. Investigate Ophthalmology \& Visual Science, v. 42, n. 6, p. 1126-1133, 2001. Disponível em: <http://www.iovs.org/cgi/reprint/42/6/ 1126.pdf > . Acesso em: 24 jul. 2008.

12 DE NATALE, R.; DRAGHI, E.; DORIGO, M. T. How prostaglandins have changed the medical approach to glaucoma and its costs: an observational study of 2228 patients treated with glaucoma medications. Acta Ophthalmologica Scandinavica, v. 82, n. 4, p. 393-396, 2004. Disponível em: < http:// www3.interscience.wiley.com/cgi-bin/fulltext/ 118808657/PDFSTART > . Acesso em: 24 jul. 2008.

13 MAIHOFNER, C.; SCHÖTER-SCHREHARDT, U.; GÜRING. H. U.; ZEILHOFER, H. U.;NAAUMANN, G. O. H.; PAHL, A.; MARDIN, C.; TAMM, E. R.; BRUNE, $K$. Expression of cyclooxygenase-1 and -2 in normal and glaucomatous human eyes. Investigative Ophthalmology and Visual Science, v. 42, n. 11, p. 2616-2624, 2001. Disponível em: <http://www.iovs.org/cgi/reprint/42/ 11/2616>. Acesso em: 24 jul. 2008.

14 STREPPA, H. K.; JONES, C. J.; BUDSBERG, S. C. Cyclooxygenase selectivity of nonsteroidal antiinflammatory drugs in canine blood. American Journal of Veterinary Research, v. 63, n. 1, p. 91-94, 2002.

15 TEIXEIRA, M. W. Dor em pequenos animais. Revista do Conselho Federal de Medicina Veterinária, n. 34, p. 31-41, 2005.

16 GARROTT, H. M.; WALLAND, M. J. Clinical case notes: glaucoma from topical corticosteroids to the eyelids. Clinical \& Experimental Ophthalmology, v. 32, n. 2 , p. $224-226,2004$

17 HERRING, I. P.; HERRING, E. S.; WARD, D. L. Effect of orally administered hydrocortisone on intraocular pressure in nonglaucomatous dogs. Veterinary Ophthalmology, v. 7, n. 6, p. 381-384, 2004.

18 LANGMAN, M. J. S.; LANCASHIRE, R. J.; CHENG, K. K.; STEWART, P.M. Systemic hypertension and glaucoma: mechanisms in common and co-occurrence. British Journal of Ophthalmology, v. 89, n. 8, p. 960963, 2005

19 RAIZMAN, M. Corticosteroid therapy of eye disease. fifty years later. Archives of Ophthalmology, v. 114, n. 8, p. 1000-1001, 1996.

20 RUTGEERTS, P. J. Review article: the limitations of corticosteroid therapy in Crohn's disease. Alimentary Pharmacology and Therapeutics, v. 15, n. 10, p. 15151525, 2001

21 RENFRO, L.; SNOW, J. S. Ocular effects of topical and systemic steroids. Dermatologic Clinics, v. 10, n. 3, p. 505-512, 1992.

22 LAM, D. C; FAN, D. S. P; NG, J. S. K.; YU, C. B. O.; WONG, C. Y; CHEUNG, A. Y. K. Ocular hypertensive and anti-inflammatory responses to different dosages of topical dexamethasone in children: a randomized trial. Clinical And Experimental Ophthalmology, v. 33, n. 3, p. 252-258, 2005.

23 JOHNSON, D.; JOHANNES, G.; FLÜGEL, C.; HOFFMANN, F; FUTA, R.; LÜTJEN-DRECOLL, E.. Ultrastructural changes in the trabecular meshwork of human eyes treated with corticosteroids. Archives of Ophthalmology, v. 115, n. 3, p. 375-383, 1997.

24 GEROMETTA, R.; PODOS, S. M.; CANDIA, O. A.; WU, B.; MALGOR, L. A; MITAGG, T. ; DANIAS, J. Steroid-induced ocular hypertension in normal cattle. Archives of Ophthalmology, v. 122, n. 10, p. 14921497, 2004. Disponível em: < http://archopht.amaassn.org/cgi/reprint/122/10/1492.pdf > . Acesso em: 24 jul. 2008.

25 CHEN, C. L.; GELATT, K. N.; GUM, G. G. Serum hydrocortisone (cortisol) values in glaucomatous and normotensive Beagles. American Journal of Veterinary Research, v. 41, n. 9, p. 1516-1518, 1980.

26 GELATT, K. N.; MACKAY, E. O. The ocular hypertensive effects of topical $0,1 \%$ dexamethasone in beagles with inherited glaucoma. Journal of Ocular Pharmacology and Therapeutics, v. 14, n. 1, p. 57-66, 1998.

27 ZHAN, G. L.; MIRANDA, O. C.; BITO, L. Z. Steroid glaucoma: corticosteroid-induced ocular hypertension in cats. Experimental Eye Research, v. 54, n. 2, p. 211218, 1992.

28 TASAKA, A. C. Antiinflamatórios não-esteroidais. In: SPINOSA, H. S.; GÓRNIAK, S. M.; BERNARDI, M. 
M. Farmacologia aplicada à medicina veterinária. 2 . ed. Rio de Janeiro: Guanabara-Koogan, 1999. p. 212226.

29 MONTOYA, L.; AMBROS, L.; KRELL, V.; BONAFINE, R.; ALBARELLOS, G.; HALLU, R.; SORACI, A. A pharmacokinetic comparison of meloxicam and ketoprofen following oral administration to healthy dogs. Veterinary Research Communications, v. 28, n. 5, p. 415-428, 2004.

30 GIULIANO, E. A. Nonsteroidal Anti-inflammatory drugs in veterinary ophthalmology. Veterinary Clinics of North America: Small Animal, v. 34, n. 3, p. 707723, 2004.

31 WINER, B. J. Statistical principles in experimental design. 2. ed. New York: McGraw-Hill, 1970. 928 p.

32 GELATT, K. N.; MACKAY, E. O. Distribution of intraocular pressure in dogs. Veterinary Ophthalmology, v. 1, n. 2/3, p. 109-114, 1998.

33 GELATT, K. N.; GUM, G. G. Inheritance of primary glaucoma in the beagle. American Journal of Veterinary Research, v. 42, n. 10, p. 1691-1693, 1981.

34 HEAT, G. Ocular therapeutic case studies: medical management of glaucoma. Optometry Today, 2002.
6 p. Disponível em: <http://www.optometry. c o. u k / a r t i c l e s / d o c s / afc0987a3c71c842159bb92a95f4634c heath 20020726.pdf>. Acesso em: 24 jul. 2008.

35 SLATTER, D. Glaucoma. In: SLATTER, D. Fundamentos de oftalmologia veterinária. São Paulo: Roca, 2005. p. 378-407.

36 RICKETTS, A. P.; LUNDY, K. M.; SEIBEL, S. B. Evaluation of selective inhibition of canine cyclooxigenase 1 and 2 by carprofen and other nonsteroidal anti-inflammatory drugs. American Journal of Veterinary Research, v. 59, n. 11, p. 1441-1446, 1998.

37 ALENCAR, M. M. A.; PINTO, M. T.; OLIVEIRA, D. M.; PESSOA, A. W. P.; CÂNDIDO, I. A.; VIRGÍNIO, C. E.; COELHO, H. S. M.; ROCHA, M. F. G. Margem de segurança do meloxicam em cães: efeitos deletérios nas células sanguíneas e trato gatrintestinal. Ciência Rural, v. 33, n. 3, p. 525-532, 2003.

38 MARTINS, B. C.; VICENTI, F. A. M.; LAUS, J. L. Síndrome glaucomatosa em cães - parte 1. Ciência Rural, v. 36, n. 6, p. 1952-1958, 2006.

39 RIBEIRO, A. P.; MARTINS, B. C.; LAUS, J. L. Síndrome glaucomatosa em cães - Parte 2. Ciência Rural, v. 37, n. 6, p. 1928-1935, 2007. 